\title{
Clinical Application of the Reverse Puncture Technique for Total Laparoscopic Colorectal Resection with Natural Orifice Specimen Extraction
}

\section{Qi Kong ( $\sim$ Kongqi1989@aliyun.com )}

Yijishan hospital of Wannan Medical College https://orcid.org/0000-0002-1182-0288

\section{Yabin Xia}

the first affiliated yijishan hospital of wannan medical college

Hu Hao

THE FIRST AFFILIATED YIJISHAN HOSPITAL OF WANNAN MEDICAL COLLEGE

\section{Yan Jina}

the first affiliated yijishan hospital of wannan medical college

\section{Longchao Wu}

the first affliliated yijishan hospital of wannan medical college

Li Xu

the first affliated yijishan hospital of wannan medical college

Xiaoxu Huang

THE FIRST AFFILIATED YIJISHAN HOSPITAL OF WANNAN MEDICAL COLLEGE

\section{Research}

Keywords: total laparoscopic colorectal resection, natural orifice specimen extraction surgery, reverse puncture technique, feasibility, safety

Posted Date: February 19th, 2020

DOI: https://doi.org/10.21203/rs.2.23956/v1

License: (a) (i) This work is licensed under a Creative Commons Attribution 4.0 International License. Read Full License 


\section{Abstract}

Objective

To explore the feasibility, safety and short-term effects of the reverse puncture technique for total laparoscopic colorectal resection with natural orifice specimen extraction surgery (NOSES).

Materials and Methods

The clinical data of 28 patients undergoing total laparoscopic anterior resection with NOSE from July 2016 to July 2019 were retrospectively analysed. The operation was performed following the principle of total mesorectal excision. The reverse puncture technique was used to put the anvil into the proximal sigmoid colon. Then, intra-abdominal anastomosis of the proximal colon and distal rectum was carried out, and the short-term efficacy was evaluated.

Results

All 28 patients successfully underwent surgery, and the average operative time was 186 (160 220) min. The average time of anvil placement was $15(12 \sim 18) \mathrm{min}$, the blood loss volume ranged from 60 to 150 $\mathrm{ml}$, and the average length of hospital stay was 9.2 (7-11) d. All of the distal resection margins were confirmed to be negative by the pathological results. No surgery-associated complications, such as anastomosis stricture, leakage, or bleeding, were observed, and no local recurrence or metastasis occurred after 6 months of follow-up.

Conclusion

Reverse puncture for total laparoscopic colorectal resection with NOSE can avoid purse-string sutures under laparoscopy and avoid the auxiliary incision at the abdominal wall, which minimizes pain after operation. This approach is safe and feasible with a shortened hospital stay and rapid recovery, which results in good short-term clinical outcomes.

\section{Introduction}

In 1991, a Japanese professor named Kitano performed the world's first laparoscopic radical surgery, which was quickly applied in the surgical treatment of colorectal tumours, becoming the mainstream minimally invasive surgical treatment ${ }^{[1]}$. Studies have shown that the short-term efficacy of laparoscopic colorectal surgery is better than that of traditional open surgery, and the long-term efficacy is no different from that of open surgery ${ }^{[2-4]}$. However, conventional laparoscopic surgery requires an auxiliary incision in the abdominal wall to complete specimen removal and digestive tract reconstruction. This auxiliary incision can cause postoperative pain, increase the risk of incision complications, affect the cosmetics of the abdominal wall, and even bring long-term adverse psychological suggestions to the patient; therefore, how to achieve better minimally invasive results and retain anal function has become a topic of focus. Recently, the trans-anal natural orifice specimen extraction (NOSE) technique, which makes a complete 
laparoscopic operation without abdominal incision possible, has been widely used in laparoscopic

colorectal surgeries ${ }^{[5-6]}$. However, how to place the anvil in the proximal sigmoid colon is a technical difficulty in alimentary canal reconstruction. We used reverse puncture technology to perform 28 total laparoscopic radical resections of colorectal cancer with satisfactory clinical effects between June 2016 and June 2019. The report is as follows.

\section{Materials And Methods 2.1 Patient data}

Twenty-eight patients ( 15 males and 13 females) with colorectal cancer underwent laparoscopic radical resection of colorectal cancer at our department between June 2016 and June 2019. The patient ages ranged from 46 to 78 years (mean: $59.8 \pm 9.8$ years). The body mass index (BMI) ranged from $18.6 \sim$ $28.4 \mathrm{~kg} / \mathrm{m}^{2}$ (mean: $21.8 \pm 9.8 \mathrm{~kg} / \mathrm{m}^{2}$ ). All patients were confirmed by colonoscopy and a pathology examination before the operation. The distance between the lowest margin of the tumours and the dentate line was 4.0-20 cm (4 $5 \mathrm{~cm}$ in 5 patients, $5 \sim 20 \mathrm{~cm}$ in 23 patients). There were 3 cases of moderately differentiated adenocarcinoma and 25 cases of poorly differentiated adenocarcinoma. All patients were examined with pelvic magnetic resonance imaging (MRI) or computed tomography (CT) to assess the tumour stage, in which 7 tumours were T1NOMO, 11 were T2NOMO, 4 were T2N1M0 and 6 were T3NOMO. There were 4 cases of diabetes mellitus and 5 cases of hypertension before the operation.

Patient inclusion criteria were as follows: $\otimes$ tumour diameter $<5 \mathrm{~cm}$ and tumour invasion less than half of the circle of the intestinal wall; $\otimes$ neoadjuvant radiotherapy and chemotherapy were not performed before the operation; $₫$ pelvic MRI showed that the tumour did not invade the surrounding tissue, and the stage was no more than T3; and $\nabla$ chest and abdominal CT showed no distant organ metastasis.

\subsection{Surgical procedure}

Before the operation, full communication with the patients' family members and signing of informed consent were needed. Routine intestinal preparation included oral antibiotics 3 days before the operation and oral magnesium sulfate 1 day before the operation. After successful anaesthesia, the patient was placed in a modified lithotomy position. The pneumoperitoneum pressure was $12-14 \mathrm{mmHg}(1 \mathrm{mmHg}=$ $0.133 \mathrm{kPa}$ ). After confirming that the tumour could be resected, the operation was performed according to the principle of total mesothelial excision (TME). The adipose tissue and lymph nodes adjacent to the mesenteric artery root were excised. Under the left mesenteric artery, the inferior mesenteric artery and vein were clamped and cut [Figure 1]. The loose tissue space around the rectum was separated, and the anal-caudal ligament was cut on the posterior wall of the rectum. Attention was paid to protect the ureteral tracts and pelvic autonomic nerve plexus. After the rectum was fully dissociated between the two layers of fascia in the pelvic visceral wall, the rectum was closed and cut with an endoscopic linear cutter $10 \mathrm{~cm}$ away from the upper edge of the tumour. 
The method of specimen removal depended on the distance between the tumour and the dentate line. When the inferior margin of the tumour was less than $5.0 \mathrm{~cm}$ from the dentate line, trans-anal pull-out resection was performed. The assistant disinfected the perineum, fully expanded the anus, and clamped the proximal end of the rectum with oval forceps through the anus [Figure 2]. The tumour-bearing intestinal canal and mesentery were slowly pulled out through the anus, repeatedly disinfected with iodine, and rinsed with distilled water. The distant rectum was closed with a curved rectal stapler at a distance of more than $2 \mathrm{~cm}$ from the lower edge of the tumour, and the specimen was removed [Figure 3]. The distal rectum was resent into the pelvic cavity, waiting for anastomosis. In cases where the lower edge of the tumour was more than $5.0 \mathrm{~cm}$ away from the dentate line, the specimens were taken through the anus after resection, and the intestines were ligated with sterile gauze strips at the top and bottom of the distal and proximal bowel incision lines, respectively. The anus was fully expanded, the anus and bowel were rinsed with dilute iodophor water, the distal bowel was cut along the pre-cut line with an ultrasonic knife, the completely removed specimen was placed in a sterile specimen bag, and the distal bowel cavity and anus were disinfected with iodophor once more. Oval forceps were used to remove the specimen bag through the anus.

The reverse puncture technique was used to insert the anvil. A $4 \mathrm{~cm}$ long 2 - 0 silk thread was placed in front of a 29 stapler (end-to-end stapler, Johnson \& Johnson Company) in advance, the trans-anal or main operation hole was used to deliver the anvil into the abdominal cavity, and an ultrasonic knife was used to cut the anterior wall of the proximal sigmoid longitudinally approximately $2 \mathrm{~cm}$. The assistant reversely pulled the thread fixed at the end of the stapler seat in the opposite direction, reserving a gap of approximately $0.5 \mathrm{~cm}$ where the thread proceeded. An Endo-Cutter (Ethicon or Medtronic) was used to close the intestinal stump, pull the anvil out of the reserved gap with the wire, and complete the insertion of the anvil in the cavity [Figure 4].

\section{Results}

All patients in this group successfully underwent laparoscopic trans-anal pelvic anastomosis, and none of them converted to open surgery. prophylactic ileostomy was conducted in 4 patients with diabetes, and closed after 3 months after surgery. The operation time was 160-220 min (average $186 \mathrm{~min}$ ), the anvil placement time was 12-18 min (average $15 \mathrm{~min}$ ), intraoperative blood loss was 60-150 ml (average $100 \mathrm{ml}$ ), and the postoperative hospital stay was $7-11$ days (average 9.2 days). No anastomotic leakage, stenosis, bleeding or other complications occurred. The distal margin of the resected specimen was negative. The postoperative pathological TNM stage was as follows: 6 patients in stage I, 17 patients in stage II, and 5 patients in stage III. The number of postoperative pathological lymph nodes was 5-24, with an average of 15.5. All the patients were treated with the FOLFOX regimen for 6 cycles. After 6 months of follow-up, no anastomotic recurrence, puncture hole implantation or distant metastasis was found.

\section{Discussion}


Compared with traditional open surgery, laparoscopic-assisted radical colorectal cancer surgery has the advantages of clear anatomical levels, less trauma, and fast recovery, while the effectiveness and safety of the operation are not significantly different from those of open surgery ${ }^{[2-4]}$; therefore, it has been widely accepted and developed by the majority of clinical workers. However, laparoscopic-assisted radical surgery requires an auxiliary incision of approximately $5 \mathrm{~cm}$ in the abdomen to remove the tumour, and an anvil is placed to complete digestive tract reconstruction. This may increase not only patient trauma but also the risk of incision infections, hernia or tumour implantation and metastasis ${ }^{[7-8]}$. In 2008 , Palanivelu ${ }^{[9]}$ reported the first laparoscopic colorectal cancer surgery performed with the NOSE technique, which made it possible to complete laparoscopic radical resection of colorectal cancer without an abdominal incision.

Specimens can be obtained through two natural lumens of the rectum or vagina, and trans-anal removal is more physiological because it does not increase trauma ${ }^{[6]}$. At present, there are three ways to remove the specimen ${ }^{[6,10]}$ : eversion resection, pull-out resection and pulling out after resection. Each method has its own operating characteristics and skills. The optimal method used to remove the specimen can be determined by the operator according to the location and size of the tumour, the degree of patient obesity and the method used to reconstruct the digestive tract ${ }^{[11-12]}$. We realized that during the operation of sphincter preservation for low and ultra-low rectal cancer, due to the limited space of the pelvic floor, it is difficult to cut and close the distal bowel of the tumour at the ideal position at one time due to the angle of the instrument, so it is difficult to ensure that the undercut margin is enough; moreover, this may increase the risk of anastomotic leakage after the operation. Therefore, if the distance between the lower edge of the tumour and the dentate line is less than $5.0 \mathrm{~cm}$, the specimen can be pulled out and resected under direct vision, which can ensure the resection of enough distal intestine and reduce the risk of tumour recurrence and implant metastasis. If the distance between the lower edge of the tumour and the dentate line is more than $5.0 \mathrm{~cm}$, the specimen can be removed through the anus after resection. During the operation, the following points should be considered: 1 . When using eversion to pull out, the rectum should be fully free, and the rectum sacrum fascia should be removed to ensure that the distal rectum has enough free length, which is conducive to pulling out the intestine with the tumour completely ${ }^{[13]} .2$. The assistant should fully expand the anus before removing the specimen and then use oval forceps to extend through the incision of the rectal stump; with the assistance of the operator, the rectal stump should be clamped, and the distal rectum and its mesentery should be slowly and forcefully pulled to the anal side and removed from the anus. During the operation, violent pulling should be avoided to avoid tearing the bowel. 3. lodophor was used to disinfect the dragged intestine repeatedly, and then, the intestine was washed with distilled water to reduce the possibility of tumour cell exfoliation and metastasis. 4. To reduce the incidence of postoperative anastomotic leakage, preventive ileostomy is recommended for patients with low rectal cancer complicated with diabetes ${ }^{[6,12]}$.

Asepsis, tumour free and anvil placement are the three key points for completing a colorectal cancer operation. The technical difficulty resides in how to place the anvil in the proximal sigmoid colon stump safely and fix it under aseptic and tumour-free conditions; this is also the key point to consider when 
completing digestive tract reconstruction under a complete laparoscope. Reverse puncture is a new type of buttress insertion method. It was first used in laparoscopic oesophagojejunostomy and further used in laparoscopic gastric cancer and colorectal cancer ${ }^{[14-16]}$. The reverse puncture method is usually used to enter the abdominal cavity through the anus or through the main operation hole. The proximal sigmoid colon needs to be cut, which may violate the sterile operation principle. Studies have shown ${ }^{[17-18]}$ that adequate intestinal preparation before the operation and standard aseptic and tumour-free conditions during the operation can effectively avoid postoperative abdominal infections. Compared with conventional laparoscopic surgery, the probability of postoperative abdominal infections in patients treated with the NOSE technique does not increase significantly. The iatrogenic spread of tumours caused by compression during the process of removing specimens through the natural cavity has also been the focus of debate. According to literature reports ${ }^{[19-20]}$, the local tumour recurrence rate of patients after NOSE surgery is not higher than that after conventional laparoscopic surgery, and there is no literature report on patients with tumours implanted in the natural cavity after NOSE surgery. This is due to the sufficiently accurate assessment of tumour location, size, and depth of invasion using pelvic MRI or enhanced CT before surgery. A tumour diameter of more than $6 \mathrm{~cm}$, a tumourous intestinal obstruction, and tumour invasion of the serosa surface were used as the exclusion criteria for NOSE surgery ${ }^{[21]}$. In addition, many techniques have been used in clinical practice to prevent the iatrogenic spread of tumours, including the use of sterile protective devices when obtaining specimens and avoiding excessive strain on the lesions during specimen collection ${ }^{[22-23]}$. Therefore, as long as the surgical indications are strictly grasped, NOSE surgery can fully guarantee the requirements of tumour-free technology. Compared with conventional laparoscopic-assisted surgery, there is also a certain technical difficulty associated with the reverse puncture procedure to place the anvil under full laparoscopy. We realize that after a short-term learning curve, with the skilled surgical skills of the surgeon and the tacit cooperation of the assistant, the placement of the anvil can be completed quickly.

The application of the reverse puncture technique in NOSE total laparoscopic colorectal resection can simplify the operation steps. There is no auxiliary incision in the abdominal wall, so it is minimally invasive, and the occurrence of complications is not increased. The effects of laparoscopic NOSE and traditional laparoscopic radical resection of colorectal cancer on postoperative rehabilitation were compared. The results showed that the NOSE technique can significantly shorten the postoperative hospital stay and exhaust time, inhibit the release of inflammatory factors, and reduce the impact on the immune function of the body; it also has good clinical application value. Ma et al ${ }^{[24]}$ analysed 837 colorectal cancer patients in 9 clinical studies and confirmed that laparoscopic trans-anal NOSE surgery

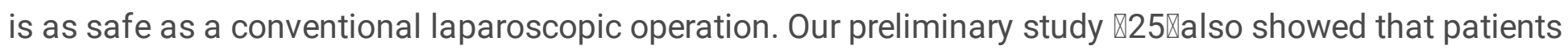
had no abdominal auxiliary incision and experienced light pain, a fast postoperative recovery, a short hospital stay, and satisfactory short-term results. No anastomotic recurrence, puncture hole implantation, or distant metastasis was found during 6 months of follow-up, which is worth further clinical application. However, due to the small sample size and short follow-up time of this group of patients, it is necessary to further verify the long-term effect through multicentre and large-scale clinical trials. 


\section{Declarations}

Acknowledgments

The authors thank the American Journal Experts Corp for their help with medical writing assistance.

Authors' contributions

QI Kong contributed to the conception and data collection of the study. YB Xia and XX Huang contributed to the provision of the study materials or patients. Y Jin and $\mathrm{H}$ Hu contributed to the data analysis and interpretation. L Xu and LC Wu drafted the manuscript. All authors read and approved the final manuscript.

Funding

This work was supported by the National Natural Science Foundation of China (NO.81902515), Wannan Medical College Cultivation Fund of the Key Scientific Project (NO. WK2014ZF13), the University Natural Science Research Project of Anhui Province (NO.KJ2008B083) , "Climbing" Cultivation Plan for the Science and Technology Innovation Team of Yanjishan Hospital of Wannan Medical College(NO.PF2019009) and Key Project of Teaching Quality and Teaching Reform Project of Wannan Medical College(NO. 2019jyxm63).

Availability of data and materials

All data are available from the corresponding author

Ethics approval and consent to participate

The authors state that this study was approved by the Ethics Committee of Yijishan Hospital at Wannan Medical College and have followed the principles outlined in the Declaration of Helsinki for all human or animal experimental investigations. In addition, informed consent has been obtained from the participants involved.

Consent for publication

All authors have given their consent for publication.

Conflicts of interest

The authors declare no conflict of interest.

\section{References}

[1] Palanivelu C, Rangarajan M, Jategaonkar PA, et al. An innovative technique for colorectal specimen retrieval: a new era of "natural orifice specimen extraction" (N.O.S.E) [J]. Diseases of the colon and 
rectum, 2008, 51(7): 1120-1124.

[2] James Fleshman, Megan Branda, Daniel J. et al. Effect of Laparoscopic-Assisted Resection vs Open Resection of Stage II or III Rectal Cancer on Pathologic Outcomes: The ACOSOG Z6051 Randomized Clinical Trial. JAMA. 2015,314(13): 1346-1355.

[3] Klein MF, Vogelsang RP. Circumferential Resection Margin After Laparoscopic and Open Rectal Resection: A Nationwide Propensity Score Matched Cohort Study.Dis Colon Rectum 2019;62: 1177-1185.

[4] Chen K, Cao G, Chen B, et al. Laparoscopic versus open surgery for rectal cancer: A meta-analysis of classic randomized controlled trials and high-quality Nonrandomized Studies in the last 5 years. Int J Surg. 2017, 39(3):1-10.

[5] Xu J $₫ X u$ LखLi L囚et al囚Comparison of outcome and side effects between conventional and transvaginal

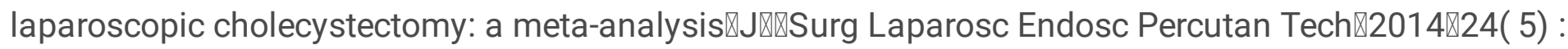
395-399凶

[6] $\mathrm{Ng} \mathrm{JL}$,Lai JH,Li HH,et al.Totally-laparoscopic versus laparoscopic-assisted low anterior resection for rectal cancer: are outcomes different?ANZ J Surg 2018;88:E818-E823.

[7] Liu Z,Efetov S,Guan X,et al.A Multicenter Study Evaluating Natural Orifice Specimen Extraction Surgery for Rectal Cancer.J Surg Res 2019;243:236-241.

[8] Shimizu H,Adachi K,Ohtsuka H,et al.Totally Laparoscopic Resection for Low Sigmoid and Rectal Cancer Using Natural Orifice Specimen Extraction Techniques.Surg Laparosc Endosc Percutan Tech 2017;27:e74-e79.

[9] Zhang H,Cong JC,Ling YZ,et al.Laparoscopic Low Anterior Resection of the Rectum with Transanal Prolapsing Specimen Extraction: Early Experience with 23 Patients.J Laparoendosc Adv Surg Tech A 2016;26:379-385.

[10] Karagul S,Kayaalp C,Sumer F,et al.Success rate of natural orifice specimen extraction after laparoscopic colorectal resections. Tech Coloproctol 2017;21:295-300.

[11] Wolthuis AM,Bislenghi G,de Buck van Overstraeten A.Transanal total mesorectal excision: Towards standardization of technique.World J Gastroenterol 2015;21:12686-12695.

[12] Morales-Conde S,Alarcón I,Yang T,et al.A Decalogue to Avoid Routine lleostomy in Selected Patients With Border Line Risk to Develop Anastomotic Leakage After Minimally Invasive Low-Anterior Resection: A Pilot Study.Surg Innov 2020;27:44-53.

[13] Shalaby M,Thabet W,Rulli F,et al.Anastomotic leakage following laparoscopic resection of low and mid rectal cancer.Ann Ital Chir 2019;90:57-67. 
[14] Hisada M,Katsumata K,Ishizaki T,et al.Complete laparoscopic resection of the rectum using natural orifice specimen extraction. World J Gastroenterol 2014;20:16707-16713.

[15] Zhao S,Zheng K,Zheng JC,et al.Comparison of totally laparoscopic total gastrectomy and laparoscopic-assisted total gastrectomy: A systematic review and meta-analysis.Int J Surg 2019;68:1-10.

[16] Efetov SK,Tulina IA,Kim VD,et al.Natural orifice specimen extraction (NOSE) surgery with rectal eversion and total extra-abdominal resection.Tech Coloproctol 2019;23:899-902.

[17] Ding Y,Li Z,Gao H,et al.Comparison of efficacy between natural orifice specimen extraction without abdominal incision and conventional laparoscopic surgery in the treatment of sigmoid colon cancer and upper rectal cancer.J BUON 2019;24:1817-1823.

[18] Zhou S, Wang X, Zhao C, et al. Comparison of short-term and survival outcomes for transanal natural orifice specimen extraction with conventional mini-laparotomy after laparoscopic anterior resection for colorectal cancer.Cancer Manag Res 2019;11:5939-5948.

[19] Wolthuis AM,Fieuws S,Van Den Bosch A,et al.Randomized clinical trial of laparoscopic colectomy with or without natural-orifice specimen extraction.Br J Surg 2015;102:630-637.

[20] Chen C,Chen H,Yang M,et al.Laparoscopy-Assisted Natural Orifice Specimen Extraction to Treat Tumors of the Sigmoid Colon and Rectum: The Short- and Long-Term Outcomes of a Retrospective Study.J Laparoendosc Adv Surg Tech A 2019;29:801-808.

[21] Guan X,Liu Z,Longo A,et al.International consensus on natural orifice specimen extraction surgery (NOSES) for colorectal cancer.Gastroenterol Rep (Oxf) 2019;7:24-31.

[22] Liu RJ,Zhang CD,Fan YC,et al.Safety and Oncological Outcomes of Laparoscopic NOSE Surgery Compared With Conventional Laparoscopic Surgery for Colorectal Diseases: A Meta-Analysis.Front Oncol 2019;9:597.

[23] Hao SZ.The application value of concept of enhanced recovery after surgery in patients with colorectal carcinoma after natural orifce specimen extraction surgery].Zhonghua Zhong Liu Za Zhi 2019;41:796-800.

[24] Ma B, Huang XZ, Gao P, et al. Laparoscopic resection with natural orifice specimen extraction versus conventional laparoscopy for colorectal disease: a meta-analysis. Int J Colorectal Dis. $2015,30(11): 1479-$ 88.

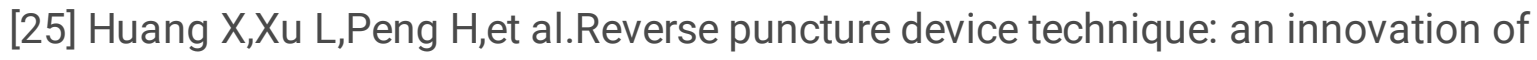
esophagojejunostomy in radical laparoscopic total gastrectomy.Future Oncol 2019;15:2807-2817.

\section{Figures}




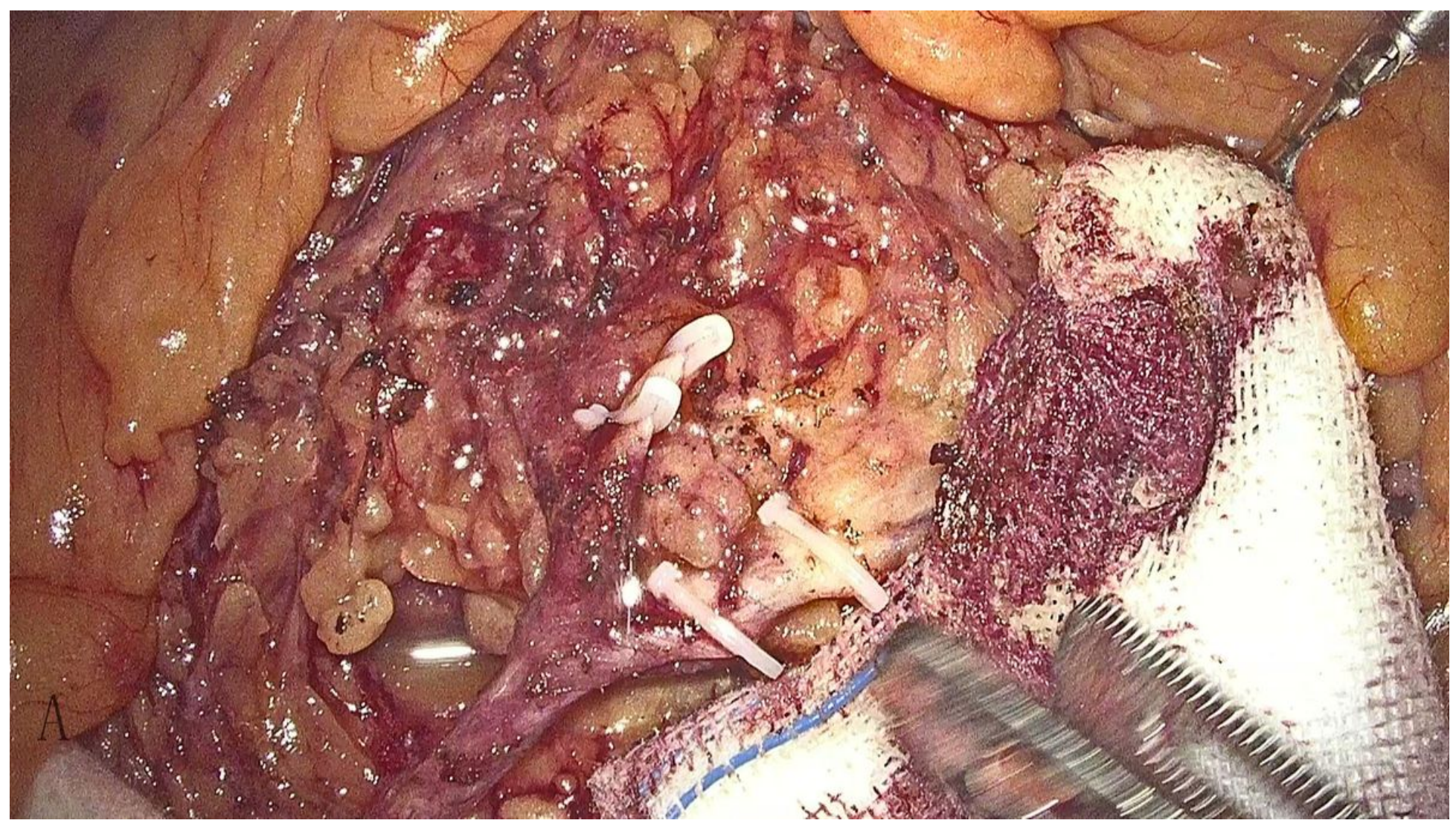

\section{Figure 1}

Total mesorectal excision with the left colonic artery preserved

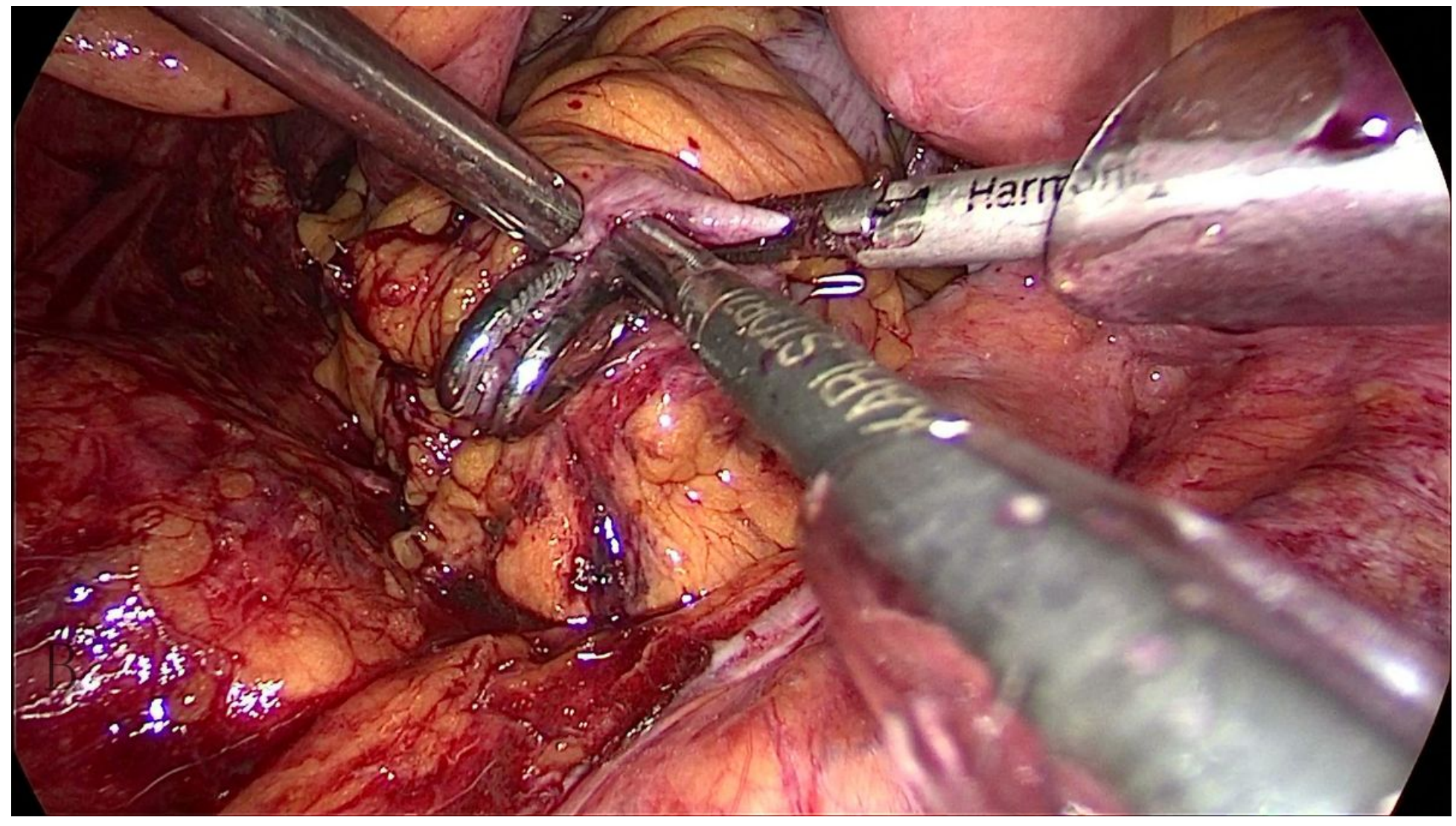


Figure 2

Pulling out the intestine and mesentery with tumours with oval forceps

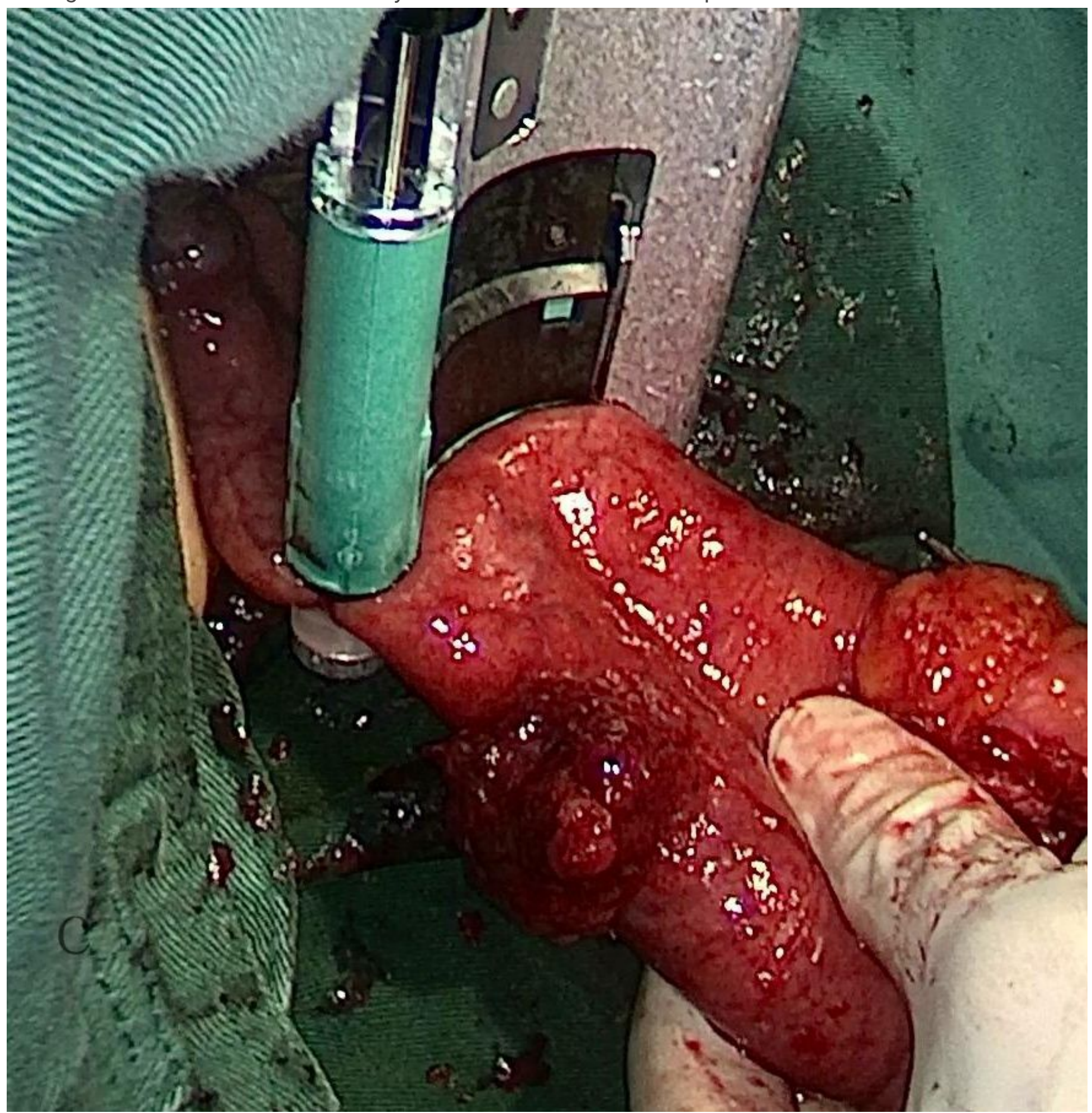

Figure 3

Specimen removed from the lower margin of the tumour 


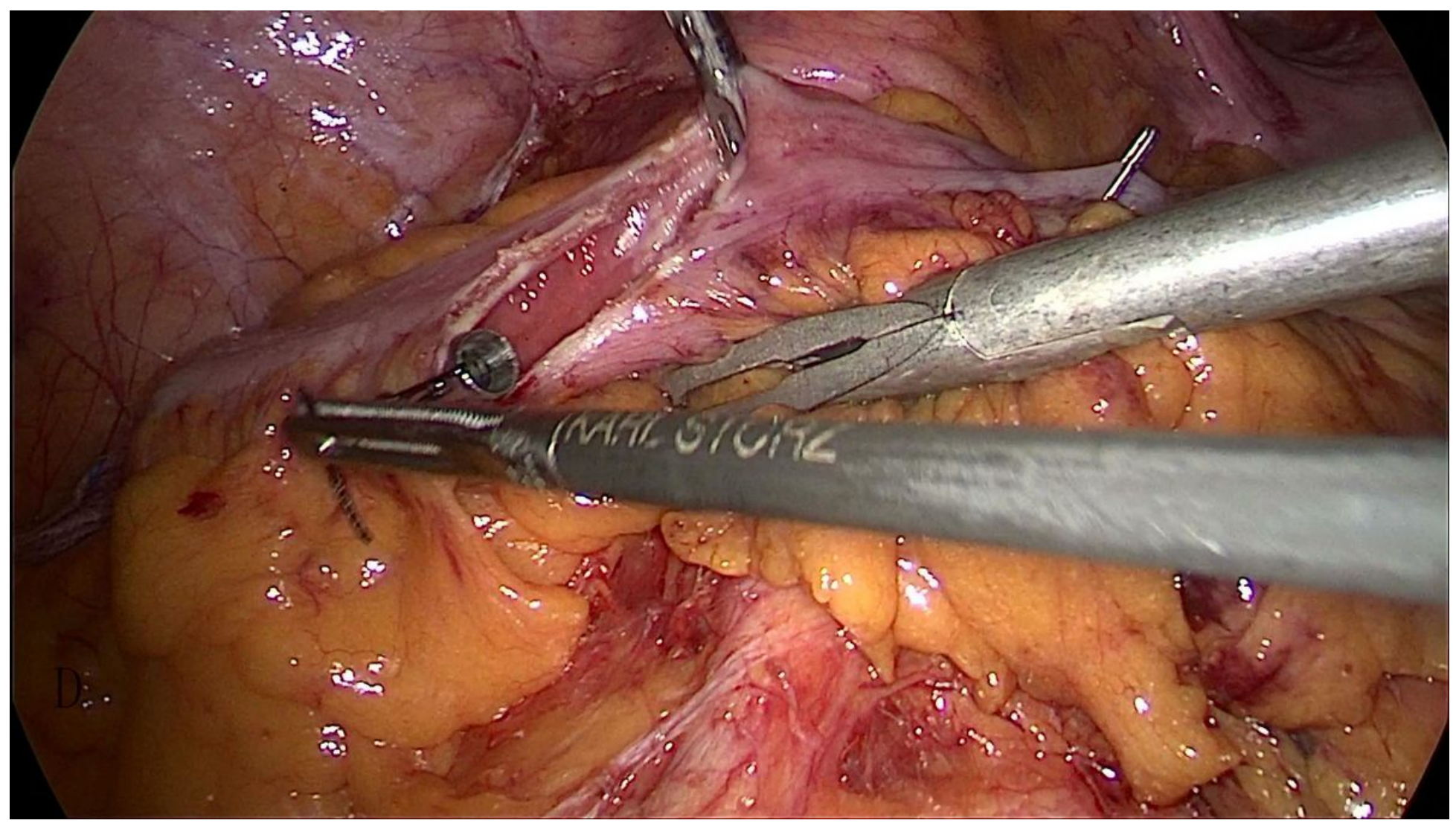

Figure 4

Inserting the anvil into the intestine with a pull thread 